\section{Andy Green}

is a co-founder and director of

The Customer Framework. His

speciality is in blending socially enabled customer management strategy with the practical design and delivery of change programmes that deliver real and sustainable financial benefit for TCF clients. A graduate in Medicinal Chemistry from UCL, Green began his career in the Airline and Travel industries. Moving into consulting, he applied his first-hand knowledge of loyalty programmes and customer management to other industry sectors - including telecoms, retail, manufacturing, financial services, CPG, pharmaceuticals and high luxury.

Keywords: data value, CRM, SCRM, Data, customer management, valuing data

Andy Green

The Customer Framework

Lily Hill House

Lily Hill Road

Ascot

Berkshire

RG12 2SJ, UK

Tel: +447876568858

E-mail: andy.green@

thecustomerframework.com

\section{Understanding the value of customer data}

\author{
Andy Green \\ Received (in revised form): 18th November, 2011
}

\begin{abstract}
That customer or consumer data have a value is universally accepted, as is the difficulty of quantifying that value. However, more organizations are finding it necessary to try. This paper provides businesses with a conceptual framework for valuing customer data in a way that can support both operational and investment decisions. It argues that this is equally possible for data-rich and data-poor organizations. It first looks at the need to understand the reasons behind the need to understand the value of customer data, and relates this to the degree of accuracy and granularity required of any valuation in order to reduce the risk of over-investment. It then proposes a framework for understanding how customer data, through the activity it enables, drives values and thus links to sustainable incremental profitability. Using this as the basis for a customer data model, it suggests an approach to attributing value to customer data at various levels from the macro 'overall value' down to the granular value of different data elements. The approach includes consideration of whether data elements are actually drivers, or merely indicators, of value. The paper concludes with suggestions on how to present data value and quality information together in a simple and compelling dashboard in order to grab the attention of senior executives and elevate consideration of customer data to the status of regular executive meeting agenda item.
\end{abstract}

Journal of Direct, Data and Digital Marketing Practice (2012) 13, 221-233. doi:10.1057/dddmp.2011.47

\section{The need for a framework for valuing customer data}

Intuitively, business leaders know that Customer Data have a value, but quantifying the value, even in broad terms relative to other assets, is something that the majority struggle with. As David Reed writes in DataIQ, Everybody agrees that data has a value. Demonstrating what that value really is and where ROI is to be found is somewhat more difficult. ${ }^{1}$ However, organizations are increasingly asking questions like, How much is a mobile phone number worth to us?; What is the missing data on our customer records costing us?; Given the cost of maintaining our own customer data, is it worth it when we can leverage data in social containers? 


\section{'Why might you want to value your Customer Data?'}

Searching online for 'how to' value customer data yields 120 million plus results. ${ }^{2}$ The more relevant results can be grouped into four broad categories:

1. How to use customer data to create value. The majority of results, typified by Pitney Bowes 2010 Thought Leadership paper, ${ }^{3}$ are devoted to how to use customer data, primarily in marketing and service, and how this adds value. They tend to start with the assumption that customer data have a value, but do not address how that value may be established.

2. Overall estimations of data value. For example, Leslie Ament of Hypatia Research quantifies the overall cost to business as a whole of poor-quality, or missing, customer data. ${ }^{4}$ And Reed, writing in dataIQ, offers some alternative ways of valuing customer data, from macro extrapolation of average profits resulting from getting data quality right to focussing on optimising costs and taxation for the business. ${ }^{5}$

3. Justifying investment in customer data. A few like Oracle's excellent 2006 paper, 'Data Management Dynamics: The ROI from Data Quality', ${ }^{6}$ provide approaches to valuing customer data for the explicit purpose of justifying investment in Customer Data Quality and/or systems.

4. The argument not to try to value customer data. Whether because it is too difficult or as Rob Karel, writing on Forrester's Blog, suggests, ... trying to place a monetary value on data and information itself is a red herring, an effort that I highly recommend all avoid - unless you enjoy philosophical exercises that don't translate to actual business value. ${ }^{7}$

What is apparent is that, with the paucity of help available, businesses would benefit from a conceptual framework for valuing customer data in a way that can support both operational and investment decisions.

\section{Be clear about why you want to place a value on your customer data}

Until you clearly define the reason for trying to place a value on your customer data, it is difficult to argue with the logic that, ... data in and of itself has no value! The only value data/information has to offer [...] is in the context of the business processes, decisions, customer experiences, and competitive differentiators it can enable. ${ }^{7}$

There are numerous occasions when being able to value data elements is of real value. The most common are probably:

- Gathering and checking customer information during inbound contacts takes time (time that could be used to cross- or up-sell) how do you justify devoting time to data at the expense of sales activity if you cannot express the value of the data being sought? 
- In today's information-rich world, many organizations try to capture all the information they can, without really considering what they need and what they can cost-justify — without understanding the value of different pieces of data, how can you realistically prioritize what should be collected?

- Approval of investments in initiatives to increase data entry consistency and/or completeness increasingly rely on being able to state the benefit - how do you quantify the return without understanding the value of the data being improved?

- How can you explain to front-line people, in a way that gets their buy-in as opposed to mandating, the importance of accurate and complete data capture if the value of it cannot be expressed?

- And how do you secure the funds to train front-line staff in data quality?

In short, without an appropriate understanding of the value of customer data, Marketers and Insight Leaders usually find themselves fighting an uphill battle to secure the necessary investment to build, maintain and optimize the data asset - unable to provide substantiation of the value the data bring in a way that Leadership Teams can understand and believe.

The problem is even greater in Consumer Packaged Goods (CPG) organizations, especially those with 'social business' ambitions. Much useful data on high value consumers and influencers are available through both traditional means and through social containers like Facebook, but the very lack of consumer data means proving the value of gathering, storing and using them is even more difficult. Even where a believable case for consumer data can be made, competition with other investment requests that promise revenue in the current quarter may result in its deprioritization. One CPG Chief Marketing Officer (CMO) even expressed it as bluntly as, 'Why waste money on a database when a fan base is more valuable, more immediate, and is a fraction of the cost?'

It does not take a crystal ball to foresee a future scenario where Facebook, Twitter, Google and the like recognize that their Brand users have abdicated responsibility for understanding their consumers. Imagine the charges they could levy if they were the sole repositories for information about your customers?

\section{What is an 'appropriate' accuracy and degree of granularity?}

The richer an organization is in customer and transactional data, the more granular and accurate the value attributed to the data can be. That is not to say that data-poor organizations cannot also attribute a value to their data, similarly, for data-rich organizations, just because you

'How accurate does my customer data valuation need to be?' can, does not mean you should!

Your reasons for wanting to value your customer data should inform both the level of accuracy and degree of granularity at which of the valuation. Without this consideration, there is a risk of over-investment 
'Create a line of sight to link customer data to business profitability'

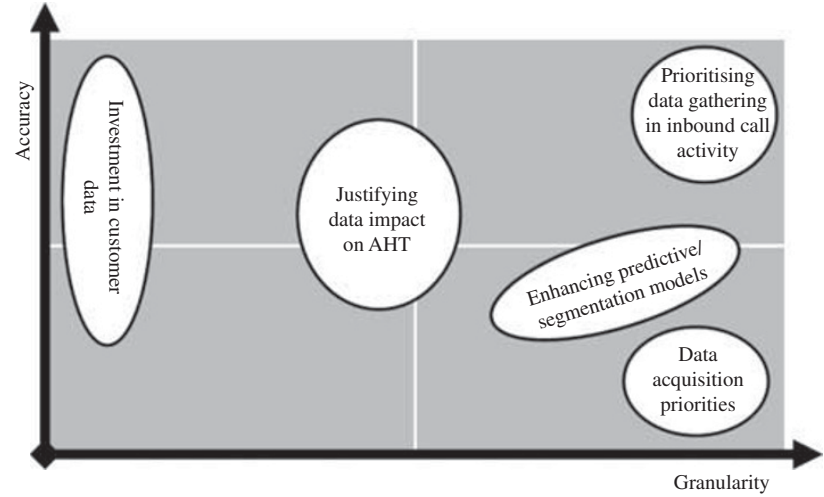

Figure 1: Different reasons for valuing data require differing levels of accuracy and granularity

attempting to establish a level of robustness beyond what is actually needed.

In Figure 1, potential different reasons for valuing customer data are plotted on axes of increasing accuracy and granularity, indicating how these different uses will require different degrees of accuracy and granularity.

As illustrated, an organization aiming to prioritize the customer information it gathers obviously needs a high degree of granularity in order to understand the relative value of different data elements.

However, on the accuracy dimension, it can probably make do with indicative, relative values and does not need to be overly robust in its valuation of each element.

Should the same organization, for example, be intending to prioritize data capture against cross-selling activity on its inbound calls, it will probably want to be much more robust in calculation of the actual value of the data elements it is targeting (ie require a high level of both accuracy and granularity in its valuation).

Conversely, an organization looking simply to justify, at a macro level, investment in customer data will need much less granularity in its calculation of value. The level of accuracy needed being a direct function of the level of anticipated challenge from the approvers of the investment.

\section{Creating the framework}

It is neither realistic nor feasible to actively collect and manage every data field that could be stored on a prospect or customer. To attempt to do so will only dilute focus and typically reduce the return on investment, or at least the measurable return. Hence, every organization should make sure that it has defined precisely what customer information it actually needs and for what purpose. If it does not already exist, this definition should be done before attempting to value the customer data.

Rob Karel's logic, namely data only has a value if it is ssed $^{7}$ is a good basis for defining the core customer information required. 
'Identify the customer data you actually need'
Start by mapping customer data to the activities it enables or supports. As first described by Neil Woodcock, Professor Merlin Stone and Bryan Foss, profit comes from customers in four ways: ${ }^{8}$

- Win - acquiring more and better quality customers

- Keep - retaining them for longer

- Develop - selling more to them

- Costs - optimizing cost of sale and service

The Schema ${ }^{\mathrm{TM}}$ model of Customer Management ${ }^{9}$ (Figure 2) shows how these four levers of value fit within the complete business system of Customer Management to deliver sustainable incremental profitability. Using a model such as this, it is possible to link customer data to activity to customer behaviour and thus, ultimately, to business profitability. This line of sight should be sufficiently robust to address the concerns expressed by Colin Rickard of DataFlux that, using a traditional marketing argument around improving customer data to get better cross- and up-sell will not cut it with Finance Directors. ${ }^{10}$

Approach the exercise to map Customer Data to the activities it supports from both ends and then take a step back and review holistically.

1. Involve the people responsible for each aspect of Win, Keep, Develop and Costs to understand the customer data they require. Do not forget that what they use today may not be what they really need, so probe around what is missing and why. Also check whether it is better to 'know' the information, or better to ask the customer at the point it is used.

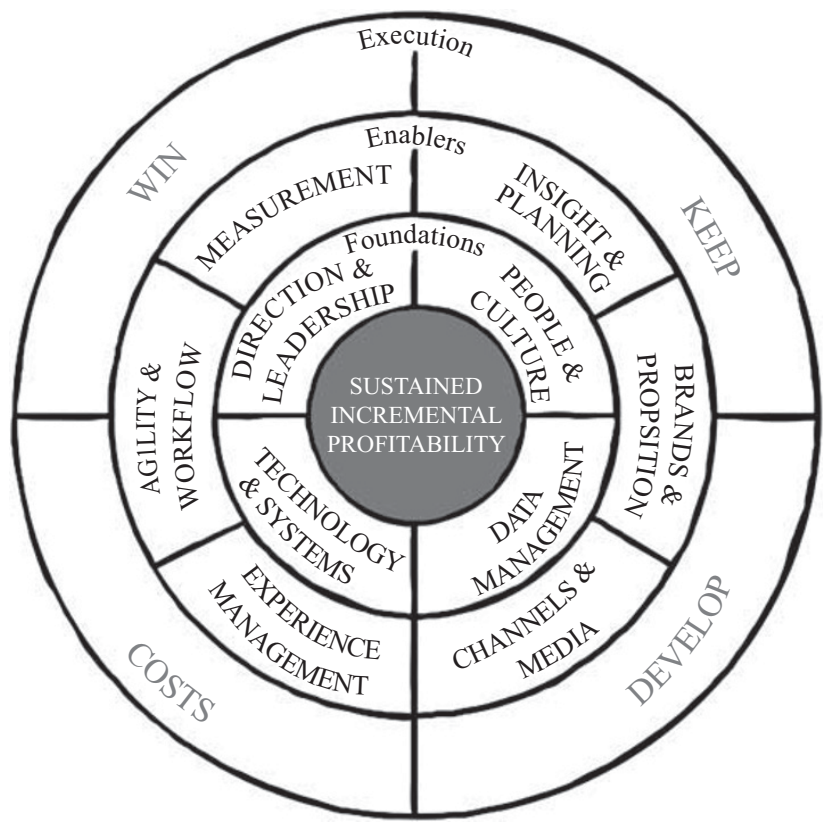

Figure 2: $\quad$ Schema ${ }^{\mathrm{TM}}-$ The business system of Customer Management 
For example, payment details may be required, but the organization may prefer not to store them and ask the customer to resupply them at each transaction.

2. Look at what information is currently gathered, created and stored, and cross-reference it with what is being used. The question why current customer data appear not to be used, or wanted. Often it will be being used, just not overtly or visibly, but be prepared for some shocks as you discover items that are gathered (or calculated) and stored, often at significant expense, and are simply not used.

This exercise alone can often add significant value. Whether it is identifying that data items commercial people say they wish they had, actually already exist and they just did not know, or finding savings from eradicating redundant data.

The output of this exercise is used to create a model similar to that in Figure 3, where each data element is fitted into the ring closest to the centre where it is first required. Each data element only appears once in the model, irrespective of how many other areas it supports.

Take a step back and consider your data model holistically. Ask yourself whether all the information needed in each area has been identified and fill in any gaps. Take care to be realistic. As discussed below, this is particularly the case in the areas of 'uniquely identify' and 'differentially serve'.

\section{Transact and get paid}

The first level in the model should contain core customer data, without which the organization cannot conduct its business. Here it is crucial to

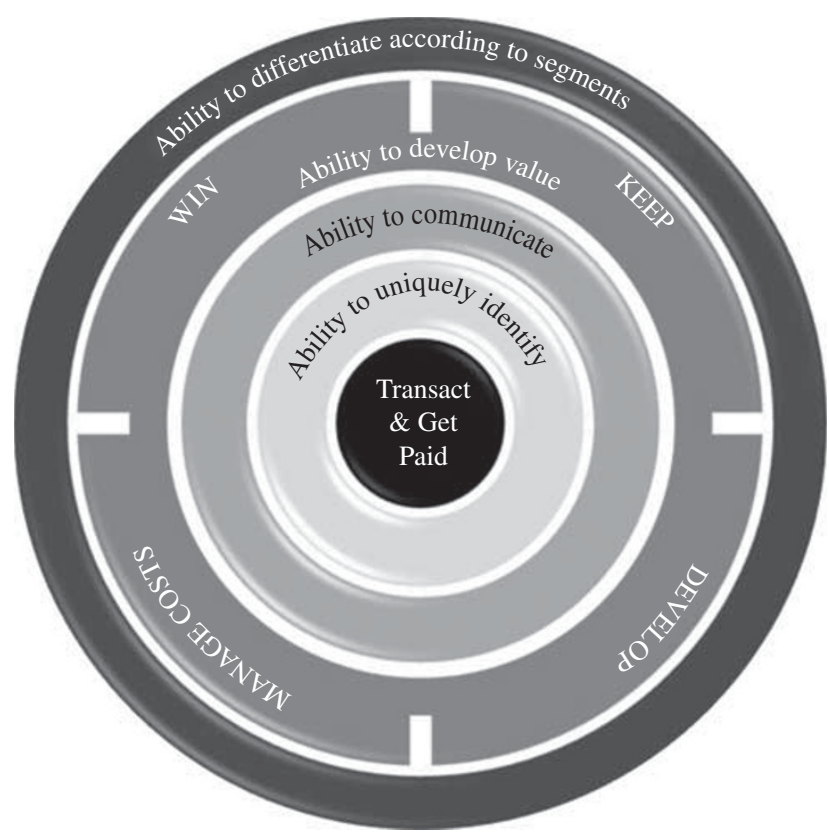

Figure 3: Data model 
ruthlessly assess what customer information is actually essential. For example, while an Insurance Company almost certainly needs the names of its customers in order to insure them, a Utility Company does not actually need the name to supply energy to a property. In fact, in many Retail organizations and almost all CPGs, there are often no data absolutely necessary to transact and receive payment.

\section{Uniquely identify}

The second level is the data that will help uniquely identify an individual, both in a customer search or identification situation and in matching, merge and deduplication activity. Be realistic about the level of accuracy you need and can hope to attain. While they may want to, most companies do not need to guarantee 100 per cent accuracy.

For example, a National ID number or Passport number is particularly accurate, but how many companies have the luxury of being in a position to ask for them without serious risk of damaging the relationship with the customer? Other data items (eg Loyalty Programme membership number) may be ideal to uniquely identify customers, but may be of insufficient penetration into the base to be viable.

In many countries, Mobile Phone number is also particularly unique to an individual and email address is increasingly 'individual' (although in many developing markets, both these may be too volatile to be reliably used in unique identification). These are data elements many more companies can reasonably ask for and use to uniquely identify their customers.

\section{Communicate}

The third level is the most important information necessary, that has not already been defined in levels 1 or 2 , in order to be able communicate with customers. At this stage, it does not necessarily need to include contact details for all channels. Often enabling communication through the single most important channel will be judged as sufficient.

This is one level where adjustment may be made once 'value' has been calculated. For example, an organization's marketing function may start by saying that email is the primary communication channel and thus place 'email address' in this level, leaving phone numbers and physical address for subsequent levels. However, work on calculating the relative value of the different 'contact' data elements may identify a different channel (and therefore data element requirement) as more valuable.

\section{Developing value}

The next level is home of the data not already covered that enable, drive and support the activity the organization undertakes to win, keep and develop its customers and to manage the cost of sale and service. 


\section{'I only really use external data, is this still relevant?'}

\section{'Are you valuing what you do or what you want to do?'}

It includes the data required to:

- understand and target acquisition and win back activity;

- understand customer tenure and to trigger and execute retention activity;

- identify and drive the main cross- and up-selling opportunities available to the majority of customers;

- understand and manage sales and service cost drivers, debt risk and recovery, and self service.

\section{Differentiate by segment}

The outermost ring houses any additional customer data needed to enable differentiation in sales, service and communication. Typically, these will be the variables used in the organization's segmentation model.

\section{Is it relevant to data-poor organizations?}

The obvious challenge to this model from data-poor organizations is whether it is actually relevant to them. Let us face it, sticking to the principle that value is in usage should also be applied to the approach if a costly theoretical exercise is to be avoided.

Whether or not monetary valuation of customer data is to be attempted, getting to this level of understanding is something all organizations, data rich and data poor alike can get value from and should, therefore, aspire to.

A data-poor organization, particularly those relying on social containers and anonymous research for their customer or consumer insight and targeting data, absolutely benefits from creating a framework similar to that described here. At a simple level, it identifies and frames what data are actually used and valued by its people, irrespective of its source. It also uncovers the data that marketers and sales people wish they had. At a more sophisticated level, it facilitates real consideration of customer data in relation to strategic business imperatives and can help Brands begin to prepare for the time when the data they rely on from external social sources like Facebook become less financially attractive.

\section{Calculating the value of the customer data}

As previously discussed, the level of granularity and/or accuracy of the calculation will vary depending on the purpose. A second consideration is necessary before embarking on any calculations, namely the appropriate basis for the data valuation. If data value derives from its use, only data that are actually used have any value. However, as Figure 4 shows, this is only one of four possible scenarios.

The most robust valuation (No. 1 in Figure 4) bases value only on the current uses of existing data (ie the data that are actually used and the value of the activity it is used in). 


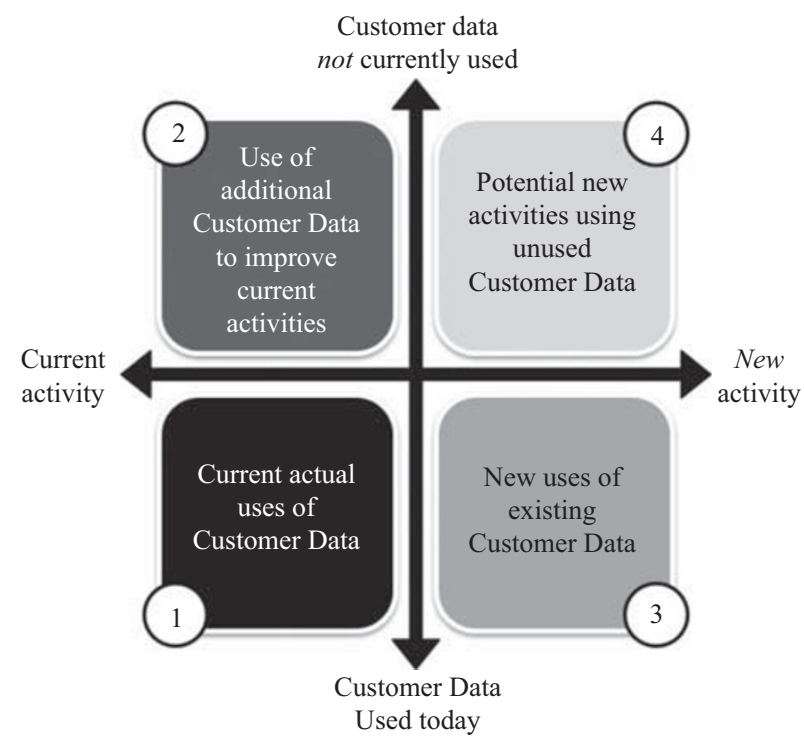

Figure 4: Different options for valuing customer data use

It may also be possible to extend the valuation to include the use of additional data to improve existing activity (Box 2 in Figure 4).

For example, marketing currently uses mobile phone numbers for outbound cross-selling (revenue increase) and enjoys better success than with fixed line home numbers. Logistics have an issue with 'undeliverable' orders and currently only use fixed line home phone numbers to call ahead to advise customers of their imminent delivery. They plan to use mobile numbers as well, but have not yet. In this scenario, it is probably reasonable to include in the valuation of mobile numbers, an uplift (cost reduction) from reduced 'undeliverable' orders. The basis for the calculation includes relative decision maker contact (DMC) rates achieved by outbound telesales. However, care must be taken not to inflate the value solely on hypothetical potential uses, or unachievable changes in customer behaviour. In this case, the difference between undeliverable rates of customers contacted and those where no contact had been possible multiplied by the increased DMC rate telesales had achieved, applied to the proportion of customers whose mobile number is known, is realistic.

Attempts to ascribe a value based on potential new uses of customer data (Box 3 in Figure 4) are more often than not going to severely compromise the robustness of the valuation unless a sound precedent to justify the attributed value can be found. For example, suggestions that include a previously unused data element in a targeting model will yield uplift in response or conversion, thus yielding incremental revenue. The suggestion may be entirely valid, but without any results, or benchmarks from similar organizations, ascribing an actual value estimate is not without risk.

The fourth quadrant, valuing currently unused data in future potential activity, can, at best, be a pure estimate (Box 4 in Figure 4). 
'Does the data element actually drive value?'
Having defined the basis for data element valuation, the next step is to take the mapping of data usage to activity and relate this to the 'value' of that activity. Depending on the degree of accuracy and robustness required, differing techniques ranging from simple calculations to complex multi-variant statistical analysis are used to create the valuations.

There is no reason that application of these concepts cannot work equally well, admittedly at differing levels of accuracy and granularity, in both data-rich and data-poor organizations. Even organizations with practically no consumer data trying to make a case to start acquiring and maintaining it could benefit from the approach by using benchmark data from other organizations to populate their business case.

\section{Value driver, or value indicator?}

A final consideration when attributing a value to individual data elements is to confirm that the data element does indeed 'drive' value.

For example, consider an airline using the stated frequency of premium class travel, where it was known, to target a business class offer. On first inspection, targeted customers where this information was available have a higher uptake of the offer than those where it was not known. However, on deeper investigation, the number of trips they say they take in a premium cabin does not actually correlate with the level of uptake. The only solid 'conclusion' is that those customers who have provided a stated frequency of premium class travel are more likely to take up the business class offer. It does not necessarily mean that the data element stated frequency of premium class travel drives value. It may well be that a customer willing to provide that information is more predisposed to the airline and therefore more likely to take up any offer. In this case, the variable is, at least in part, merely 'indicating' and not 'driving' customer value.

Your calculation of data element value needs to incorporate consideration of this dynamic.

In this respect, attempts to attribute value to marketing consent merits specific discussion.

On the one hand, it can be argued that, in most markets, without marketing consent, the organization is unable to use any customer data to drive value, and therefore the entire 'value' of its database should be attributed to consent. On the other hand, the argument is that having consent creates no value and, if misused, could in fact destroy value. Furthermore, the extent to which 'affirmative' consent indicates a greater pre-existing propensity to buy from the organization, versus the value it enables the organization, is open to debate.

The truth probably lies between the two. However, it is probably a moot point and of little value in answering because, any organization intending to use its customer data needs consent and it is unlikely that 'justification' to obtain consent status will actually be required - so why waste time and money attempting to calculate its value?

It is probably appropriate not to include marketing consent in your customer data value model. 
'How do we get around gaps in our data?'

\section{What input data are needed?}

Obviously, the richer the organization is in terms of customer behaviour data and transactional data, the more accurate and granular the output can be. In an ideal world, lifetime value, product tenure and the impact on these of different activities would be used. However, very few organizations will be in the enviable position of having all this information available and, if they do, they probably already understand and accept the value to their business of their customer data.

There are many workarounds that can be used to close the gaps and make sensible and believable assumptions where less than perfect base data are available. These range from developing proxies, seeking out less obvious sources of insight into customer data (eg risk and debt recovery can often have more useful information on the relative importance of different customer data elements than the marketing team function), to the use of external benchmark data.

However, despite all the innovative thinking employed to identify proxies and create acceptable model inputs, data-poor organizations may simply not be able to establish actual financial values for the activity they undertake. The beauty of the approach described here is that it can work with whatever metrics the organization uses to judge its return on marketing investment, not just hard financial values.

\section{Presenting and using customer data value information}

The knowledge of how valuable customer data are, whether at an aggregate, relative or detailed data element level can be extremely powerful, but only if it is presented well.

How it is presented is entirely dependent on the purpose of the valuation in the first place. However, bringing it together with data quality information to show record level and field level data quality alongside value is something that will usually grab the attention of senior leaders, particularly if it shows the value (or cost) of, for example, not being able to identify or communicate with a customer and, on the same chart, shows how many customers it is not possible to identify or communicate with.

The proportion of 'missing value' shown in the central bar of the example customer data dashboard in Figure 5 can rapidly become a constant agenda item on senior team agendas — giving customer data their due exposure as a critical driver of customer value.

\section{Conclusion}

The conceptual framework and structured methodology presented here provides a guide to organizations needing to overcome the challenges of quantifying the value of their customer or consumer data. By following the series of simple steps, it should be possible for data-rich and data-poor organizations to achieve an appropriate level of valuation that meets their particular needs. 


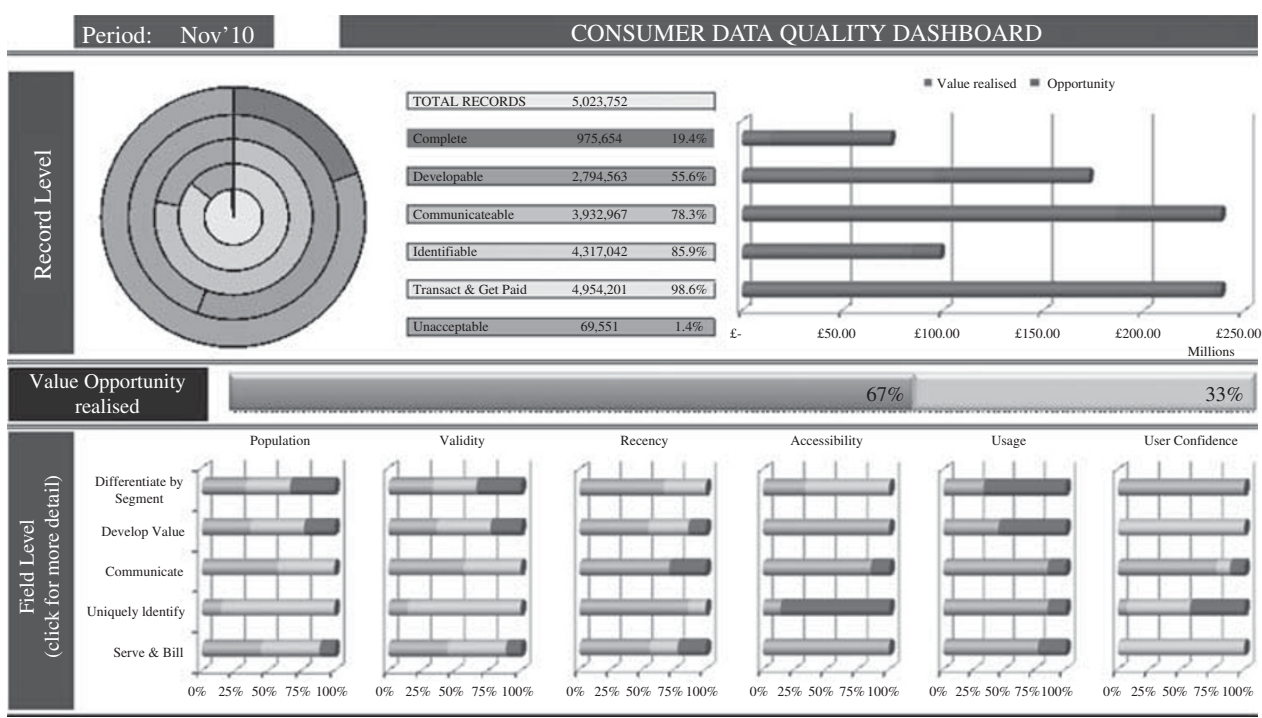

Figure 5: Data quality and value dashboard example

The concepts presented consider how:

- an initial clarity around the reasons for wanting to place a value on the data defines the level of granularity and accuracy to be sought and prevents overinvestment;

- the Schema model forms a basis on which a line of sight from customer data, through its uses, to the 4 Customer Management levers of incremental sustainable profitability;

- by organizing customer data according to the operational, marketing and analysis activities in each of these areas, it is possible to create a data model to classify the different data elements according to the roles they play;

- this structure can be used to attribute value overall and, in some cases, to individual data elements, and to discuss some of the pitfalls and limitations organizations should watch out for.

Simply mapping Customer Data to the activities it supports can often add significant value - identifying data that commercial people need, but did not realize they had and/or finding savings from eradicating redundant data. Even where monetary valuation of customer data is not required, this level of understanding is something all organizations, data rich and data poor alike, can get value from and should, therefore, aspire to.

Finally, we have seen how combining the value of customer data with data quality measurement in a simple and highly visual dashboard grabs the attention of senior executives and can raise consideration of customer data to its rightful status - as a core item on the executive team agenda. 


\section{References and Notes}

1. Reed, D. (2011) 'Cashing in on data's valuedataIQ, Autumn 2011, p. 4.

2. http://www.google.co.uk/search?hl=en\&q=how+to+value+customer+data\&meta=\&rlz=1I7ADR A_en.

3. http://www.pbinsight.eu/uk/files/download/data/MaximizingvaluefromCustomerData.pdf.

4. http://searchbusinessanalytics.techtarget.com/tip/Exploiting-the-value-of-customer-data-withanalytics.

5. Reed, D. (2011) 'Cashing in on data's valuedataIQ, Autumn 2011, p. 4-6.

6. http://www.oracle.com/us/products/applications/master-data-management/roi-from-data-quality168367.pdf.

7. http://blogs.forrester.com/rob_karel/11-03-29-stop_trying_to_put_a_monetary_value_on_data_ its_the_wrong_path.

8. Woodcock, N., Stone, M. and Foss, B. (2003) The Customer Management Scorecard, Kogan Page, Great Britain and the United States, p. 12.

9. Schema Model reproduced courtesy of The Customer Framework.

10. Reed, D. (2011) 'Cashing in on data's valuedataIQ, Autumn 2011, p. 6. 